Studien zum Wirtschaftsstrafrecht

\author{
herausgegeben von \\ Klaus Tiedemann / Bernd Schünemann
}

Band 20 
Robert Maiazza

\section{Das Opportunitätsprinzip im Bußgeldverfahren unter besonderer Berücksichtigung des Kartellrechts}

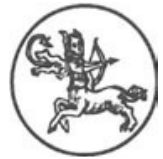

Centaurus Verlag \& Media UG 2003 
Der Autor, geb. 1972, studierte Rechtswissenschaft in Bayreuth und Freiburg im Breisgau und promovierte 2002 an der Universität Freiburg. Er ist Richter am Landgericht Berlin.

Die Deutsche Bibliothek - CIP-Einheitsaufnahme

\section{Maiazza, Robert:}

Das Opportunitätsprinzip im Bußgeldverfahren unter besonderer Berücksichtigung des Kartellrechts /

Robert Maiazza. - Herbolzheim : Centaurus-Verl., 2003

(Studien zum Wirtschaftsstrafrecht; Bd. 20)

Zugl.: Freiburg (Brsg.), Univ., Diss., 2002

$$
\text { ISBN 978-3-8255-0394-9 ISBN 978-3-86226-871-9 (eBook) }
$$

DOI 10.1007/978-3-86226-871-9

\section{ISSN 0938-9512}

Alle Rechte, insbesondere das Recht der Vervielfältigung und Verbreitung sowie der Übersetzung, vorbehalten. Kein Teil des Werkes darf in irgendeiner Form (durch Fotokopie, Mikrofilm oder ein anderes Verfahren) ohne schriftliche Genehmigung des Verlages reproduziert oder unter Verwendung elektronischer Systeme verarbeitet, vervielfältigt oder verbreitet werden.

CC CENTAURUS Verlags-GmbH \& Co. KG, Herbolzheim 2003

Satz: Inga Gottlebe Grafik-Design, Berlin Umschlaggestaltung: DTP-Studio, Antje Walter, Hinterzarten 
Meinen Eltern 


\section{Inhalt}

Abkürzungsverzeichnis.........................................................................................................

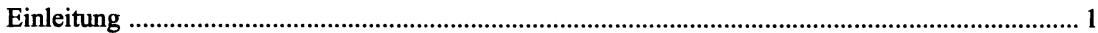

$1 \quad$ Bedeutungsgehalt und Grundlagen des Opportunitätsprinzips ...................................... 8

1.1 Opportunitätsprinzip als schwach gebundener Modus der Entscheidungsfindung.......... 11

1.2 Ermessen: Das Wesensmerkmal des Opportunitätsprinzips .......................................... 12

1.2.1 Begriff und Existenz von Ermessen .......................................................................... 13

1.2.2 Wesen und Funktion der Ermessensentscheidung ..................................................... 15

1.2.2.1 Rechtsfolgeermessen als normativ eröffnete Wahlfreiheit zwischen rechtmäßigen Handlungsalternativen ...................................................................................... 15

1.2.2.2 Tatbestandsergänzungsermessen als gesetzlicher Auftrag der Verwaltung zur

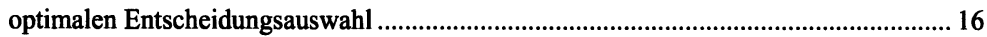

1.2.2.3 Zusammenfassende Darstellung der verwaltungsrechtlichen Ermessensfehlerlehre ....... 18

1.3 Tauglichkeit der verwaltungsrechtlichen Ermessenslehre für das

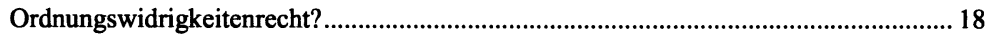

$1.4 \quad$ Opportunitätsprinzip und Beliebigkeit der Entscheidungsbildung .................................. 20

1.5 Strafrechtlicher Ermessensbegriff des Bußgeldverfahrens ...........................................2 23

$1.6 \quad$ Verfassungsrechtliche Grenzen der Ausübung von Ermessen........................................ 27

1.6.1 Die Restriktion der gleichmäßigen Gesetzesanwendung ............................................... 28

1.6.2 Die Restriktion der verhältnismäßigen Gesetzesanwendung ........................................ 29

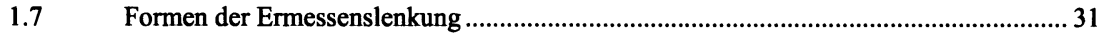

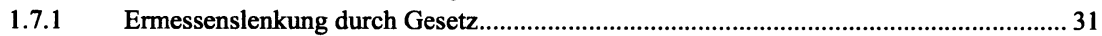

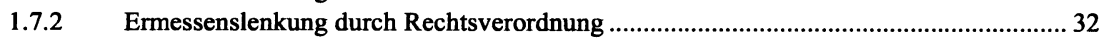

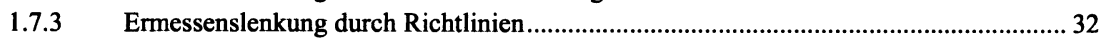

1.8 Ermessensreduzierung und subjektives Recht auf bestimmte Ermessensausübung ........ 33

2 Rechtsgeschichtliche Darstellung der Genese des Opportunitätsdenkens im

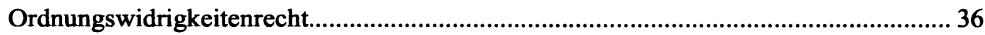

2.1 Das Polizeistrafrecht nach Inkrafttreten der StPO bis 1933 ........................................ 37

2.2 Das Opportunitätsprinzip im Verwaltungsstrafverfahren während der NS-Zeit ............. 41

2.2.1 Ermittlungs- und Verfolgungsprinzipien im Steuerstrafverfahren................................... 43

2.2.2 Ermittlungs- und Verfolgungsprinzipien im Preisstrafrecht ........................................ 44

2.2.3 Ermittlungs- und Verfolgungsprinzipien im Devisenstrafrecht ..................................... 46

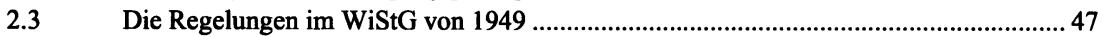

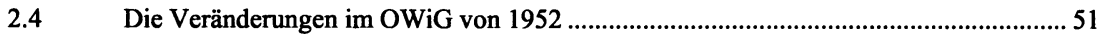

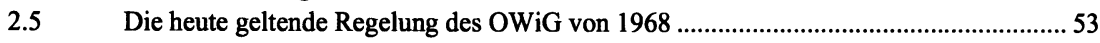

3 Systembedingungen, Defizite und Vorzüge des Opportunitätsprinzips im Vergleich zum Legalitätsprinzip............................................................................................. 57

3.1 Die allgemeine Bindung der Verfolgungsbehörden an die Rechtsauffassung der

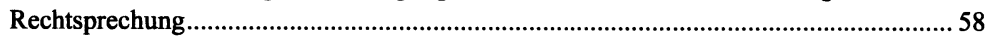

3.2 Das Legalitätsprinzip als verfahrensrechtliche Antwort auf die Anforderungen des

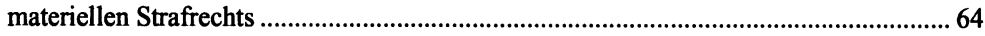

3.3 Das Verhältnis von Opportunitätsprinzip und materiellem Ordnungswidrigkeitenrecht.

3.3.1 Das der Untersuchung zugrunde gelegte Verhältnis von Straftat und Ordnungswidrigkeit . 
3.3.2 Abgeschwächter Normbefehl bei Ordnungswidrigkeiten?....

3.4 Verfahrensrechtlich geschaffene Defizite durch in das Ermessen gestellte

Sanktionsmöglichkeiten 69

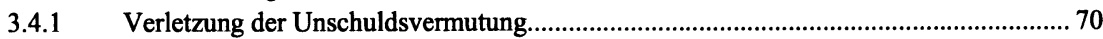

3.4.2 Mangelnde Gleichbehandlung und Willkür................................................................ 71

3.4.3 Versagung der Justizgewährung im Bußgeldverfahren .............................................. 72

3.4.4 Verletzung des gleichheitswahrenden Bestimmtheitsgrundsatzes? ................................ 74

3.5 Rechtfertigung und Abschwächung der Defizite im Bußgeldverfahren ......................... 79

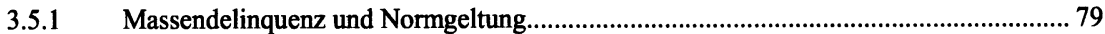

3.5.2 Verfahrensökonomie und Effizienz als überwiegende Interessen .................................. 81

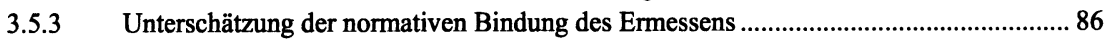

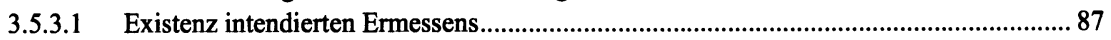

3.5.3.2 Dienstrechtliche Bindungen als Kompensation schwacher Entscheidungsvorgaben ...... 88

3.5.3.3 Strafrechtliche Absicherung pflichtgemäßer Ermessensbildung .................................. 90

3.5.3.4 Der Begründungszwang, das Anhörungsgebot und die Mitteilungspflichten als Absicherung gegen ermessenswidrige Entscheidungen..................................................93

3.5.3.5 Vorhandensein ermessenslenkender Verwaltungsvorschriften....................................... 95

3.5.4 Erhöhte Akzeptanz von Opportunitätsentscheidungen durch gesetzlich begünstigte

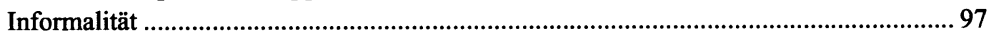

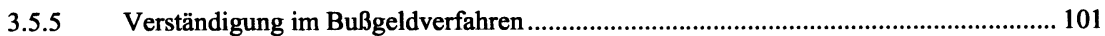

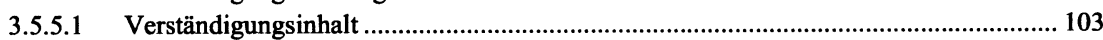

3.5.5.2 Rechtliche Wirkung der Verständigung................................................................. 103

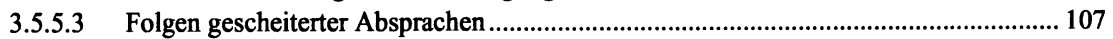

4 Fokussierung der einstellungs- und verfolgungsrelevanten Ermessensfaktoren............. 109

4.1 Ermessensbildung der Verfolgungsbehörden im Modell der allgemeinen

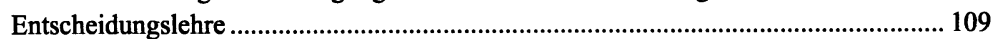

4.2 Die Ermessenskonzeption der Rechtsprechung für das Bußgeldverfahren ................... 114

4.3 In der Literatur bisher vorgeschlagene Ermessenskonzeptionen .................................. 115

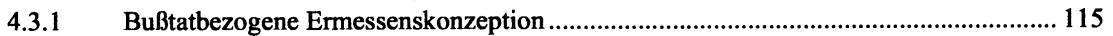

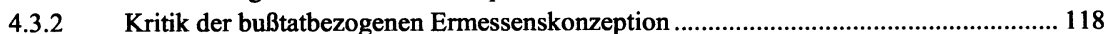

4.3.3 Legalitätsbezogene Ermessenskonzeption: Die analoge Anwendung der Opportunitätsvorschriften der StPO zur Strukturierung des negativen

Entschließungsermessens der Verfolgungsbehörde.................................................... 120

4.3.3.1 §153 Abs. 1 StPO: Einstellungsgrund: Geringe Schuld und fehlendes öffentliches

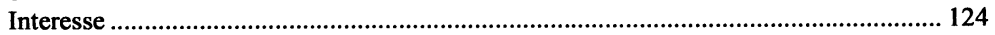

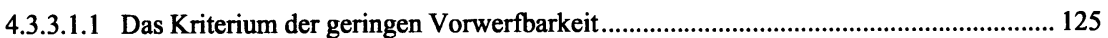

4.3.3.1.2 Das Kriterium des fehlenden öffentlichen Interesses an der Durchführung des

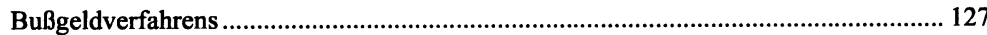

4.3.3.2 $§ 153$ a StPO: Kompensation des öffentlichen Strafinteresses durch freiwillige Auflagenerfüllung

4.3.3.3 $\S \S 153$ b StPO, 60 StGB: Einstellung wegen Selbstbestrafung ...................................... 134

4.3.3.4 $\S \S 153$ c-e StPO: Einstellungsgrund: Auslandsbezug und dessen europarechtliche

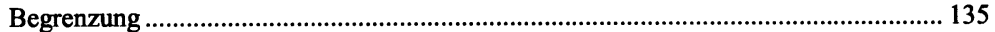

4.3.3.5 $\S \S 154,154$ a StPO, Art. 6 Abs. 1 S. 1 EMRK: Konzentrations- und Beschleunigungsgebot im Bußgeldverfahren 
4.3.3.6 $§ 154$ b StPO: Drohende oder vollzogene Abschiebung als Einstellungsgrund im Bußgeldverfahren.

4.3.3.7 §154 c StPO: Ausprägung des Wechselwirkungsverhältnisses zwischen

Legalitätsprinzip und Opportunitätsprinzip

4.3.3.8 $\S \S 45,47$ JGG: Grundlage eines allgemeinen Diversionsgedankens im Bußgeldverfahren.

4.3.3.9 $\S \S 154$ d, 154 e StPO: Der Ermessensgesichtspunkt der Vorgreiflichkeit des

Verfahrens.

4.3.3.10 Kronzeugen- und Selbstanzeigeregelungen als Ermessenskriterien in $\S 47$ Abs. 1 OWiG.

4.3.3.11 Nemo-tenetur-Grundsatz und Ermessen ..................................................................... 151

4.3.4 Zusammenfassende Würdigung der legalitätsbezogenen unteren Grenzen der Ermessensbildung.

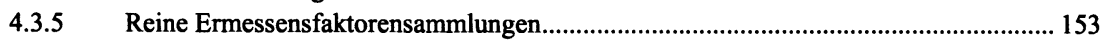

4.3.6 Kritik der bisherigen Ermessensfaktorensammlungen................................................. 154

4.4 Eigener Standpunkt: Der vom Bußgeldtatbestand intendierte Rechtsgüterschutz als Entscheidungsgrundlage der Verfolgungsbehörden und maßgeblicher Zweck der Ermessensbetätigung........................................................................................... 154

4.5 Die durch Tatbestandstypen gesetzten oberen Grenzen der Ermessensausübung ......... 155

4.5.1 Das positive Entschließungsermessen der Verfolgungsbehörden.................................. 161

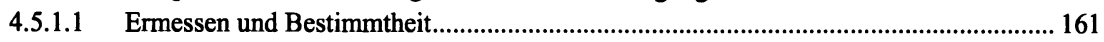

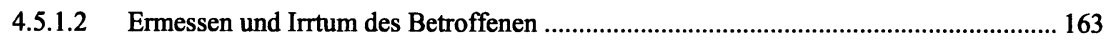

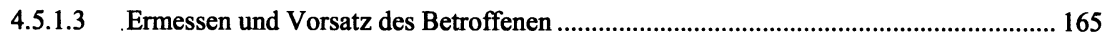

4.5.1.4 Ermessen und Rechtfertigung des Betroffenen.............................................................. 167

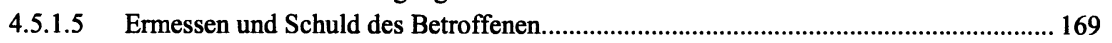

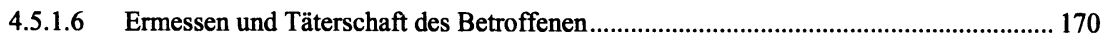

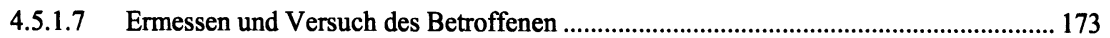

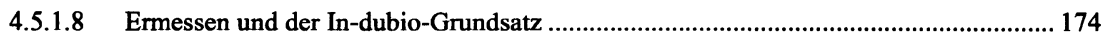

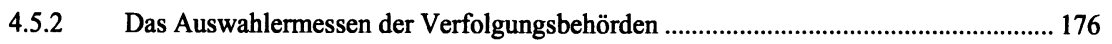

4.5.2.1 Sanktionsbezogene Entscheidungsmöglichkeiten der Verfolgungsbehörden................. 176

4.5.2.1.1 Abmahnung bzw. Verwarnung mit und ohne Verwarnungsgeld ................................... 177

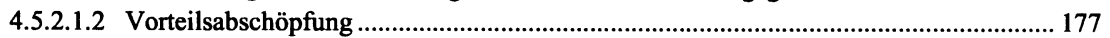

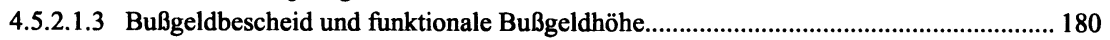

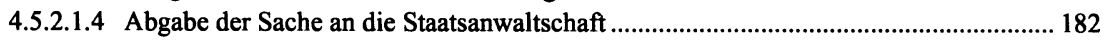

4.5.2.2 Adressatenbezogene Entscheidungsmöglichkeiten der Verfolgungsbehörden.............. 185

4.5.2.2.1 Die Bedeutung der $\S \S 9,30,130$ OWiG für das Verfolgungsermessen ......................... 186

4.5.2.2.2 Hierarchieauswahl bei der Ausübung des Verfolgungsermessens.................................. 188

4.6 Strafwürdigkeit und Strafbedürftigkeit als Bezugspunkt des

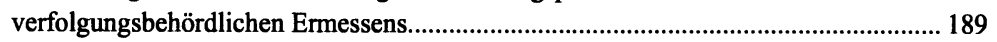

4.7 Ermessensbildung nach Einspruch des Betroffenen gegen den Bußgeldbescheid ......... 191

4.8 Ermessensbildung der Staatsanwaltschaft im Bußgeldverfahren.................................. 192

4.9 Ermessensbildung des Gerichts im Bußgeldverfahren ............................................... 193

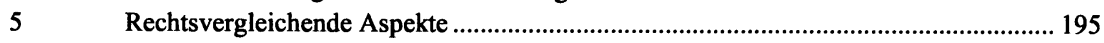

$5.1 \quad$ Die Rechtslage in den Vereinigten Staaten von Amerika ............................................ 195

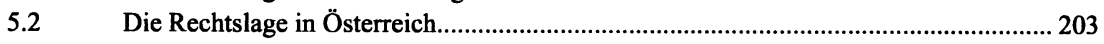

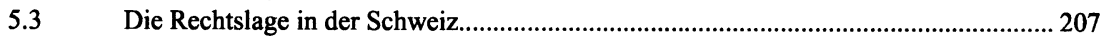




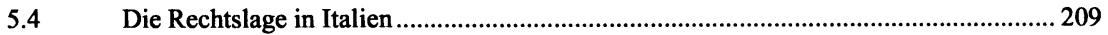

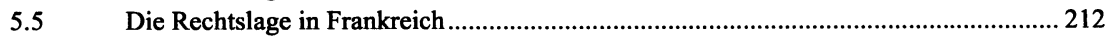

5.6 Zusammenfassende Würdigung der untersuchten ausländischen Rechtsordnungen ..... 215

6 Die Exemplifizierung des gewonnenen Entscheidungsmodells anhand schwerwiegender Ordnungswidrigkeiten.................................................................... 217

6.1 Die Bedeutung des Begriffs ,,schwerwiegende Ordnungswidrigkeit““........................... 217

6.1.1 Abgrenzungskriterien und Funktion des Begriffs bei der

Entscheidungsprogrammierung der Verfolgungsbehörden........................................ 219

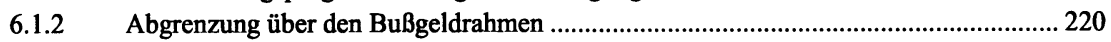

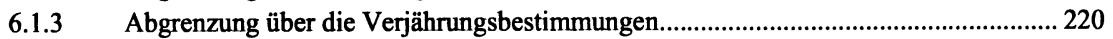

6.1.4 Differenzierung nach möglichen Opferinteressen respektive nach der Deliktsnatur..... 221

6.2 Die in ihrem Unrechtsgehalt den Straftatbeständen graduell vergleichbaren

Bußgeldtatbestände als Hauptanwendungsfälle der schwerwiegenden

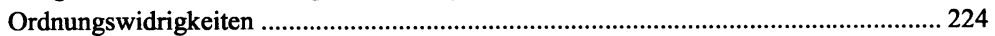

6.2.1 Die Kartelldelikte als Beispiele schwerwiegender Ordnungswidrigkeiten.................... 227

6.2.1.1 Richtigkeit der kartellrechtlichen Antipönalisierungstheorie?......................................229

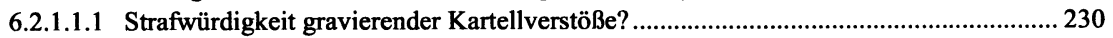

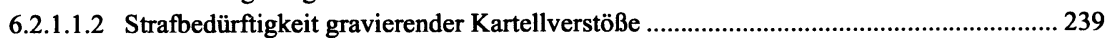

6.2.1.2 Systemkohärente Steuerung des Verfolgungsermessens ........................................... 243

6.2.1.2.1 Berücksichtigung einer, ,rule of reason“ bei der Ermessensbildung? ............................ 245

6.2.1.2.2 Die „sog. Bagatellbekanntmachung“ als Reglementierung des negativen

Entschließungsermessens bei der Verfolgung von Ordnungswidrigkeiten gem. § 81

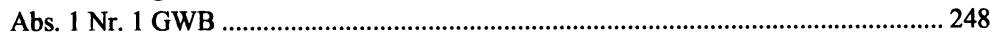

6.2.2 Positives Entschließungsermessen bei gravierenden Kartellrechtsverstößen ................. 251

6.2.2.1 Faktische Ermessensbildung des BKartA bei Verstößen gegen gesetzliche Verbote gemäß § 81 Abs. 1 Nr. 1 GWB............................................................................. 253

6.2.2.1.1 Ermessensbildung bei Zuwiderhandlungen gegen das Kartellverbot und das Verbot bestimmter Vertikalvereinbarungen.

6.2.2.1.2 Ermessensbildung bei Zuwiderhandlungen gegen das Verbot des Mißbrauchs einer marktbeherrschenden Stellung................................................................................ 258

6.2.2.1.3 Ermessensbildung bei der Zuwiderhandlung gegen das Verbot der

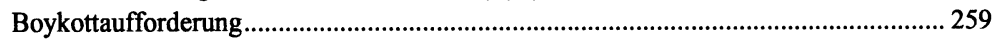

6.2.2.1.4 Ermessensausübung bei Zuwiderhandlungen gegen die Verbote der

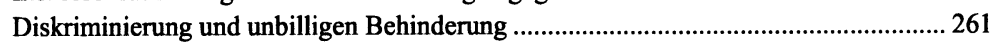

6.2.2.1.5 Exkurs: Die sich aus der Bekanntmachung des BKartA Nr. 68/2000 ergebenden

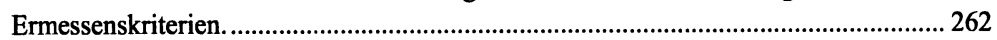

6.2.2.2 Ermessensbildung bei Verstößen gegen Auskunfts- und Meldepflichten, $\S 81$ Abs.

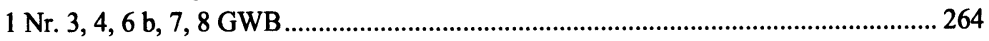

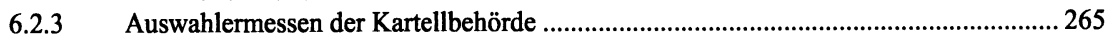

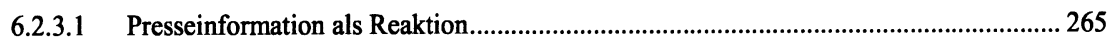

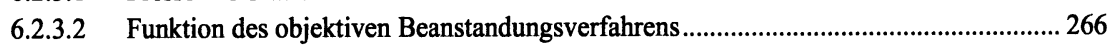

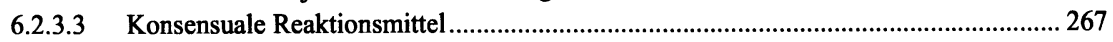

6.2.4 Ermessensbildung bei der Verfolgung strafbarer Submissionsabsprachen..................... 268

$7 \quad$ Zusammenfassung der wichtigsten Ergebnisse der Arbeit ......................................... 272

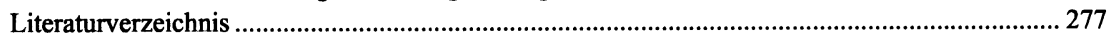




\section{Abkürzungsverzeichnis}

a.A., A.A.

aaO.

Abl.

Abs.

AD

ADM

AG

AllMBI.

Alt.

Anm.

AO

AöR

ArbZG

Art.

AStBV

AsylVfG

AT

Aufl.

AuslG

AV

AWG

$\mathrm{B} / \mathrm{K}$

BayOblG

BayStMI

BayVbl.

BB

Bd.

BFH

BG

BGB

BGB1.

BGE

BGH

BGHR

BGHSt.

BGHZ.

BHO

BJagdG

BKartA

bol andere Ansicht

am angegebenen Ort

Amtsblatt

Absatz

Antitrust Division

Antitrust Division Manual

Amtsgericht

Allgemeines Ministerialblatt der Bayrischen Staatsregierung

Alternative

Anmerkung

Abgabenordnung von 1977

Archiv des öffentlichen Rechts

Arbeitszeitgesetz

Artikel

Anweisung für das Straf- und Bußgeldverfahren (Steuer)

Asylverfahrensgesetz

Allgemeiner Teil

Auflage

Ausländergesetz

Allgemeine Verwaltungsvorschrift

Außenwirtschaftsgesetz

Kommentar zum Bonner Grundgesetz (Bonner Kommentar)

Baden-Württemberg

Bayrisches Oberstes Landesgericht

Bayrisches Staatsministerium für Inneres

Bayrische Verwaltungsblätter

Betriebsberater

Band

Bundesfinanzhof

Bundesgesetz (Schweiz)

Bürgerliches Gesetzbuch

Bundesgesetzblatt

Entscheidungen des Schweizerischen Bundesgerichts

Bundesgerichtshof

BGH-Rechtsprechung, Strafsachen

Entscheidungen des Bundesgerichtshofes in Strafsachen

Entscheidungen des Bundesgerichtshofes in Zivilsachen

Bundeshaushaltsordnung

Bundesjagdgesetz

Bundeskartellamt

base offense level 
BStP

BTDrucks.

BtmG

BVerfG

BVerfGE.

BVerwG

BVerwGE.

bzw.

Cost.

CPP

D.C.C.

d.h.

DAR

DB

ders.

DevG

DJ

DJT

DÖV

DRiZ

DStR

DStZ

DVB1.

EEC

EGStPO

EGV

Einf.

Einl.

EMRK

EuGH

EuGHE

EuZW

EWGV

ff.

FK GWB

Fn.

f.

FS

GA

gem.

GmbHG

GewArch.

GewO

GG

GK GWB
Bundesgesetz über die Bundesstrafrechtspflege (Schweiz)

Bundestags-Drucksache

Betäubungsmittelgesetz

Bundesverfassungsgericht

Entscheidungen des Bundesverfassungsgerichts

Bundesverwaltungsgericht

Entscheidungen des Bundesverwaltungsgerichts

beziehungsweise

Costituzione

Code de procédure pénal

District of Columbia Circuit

das heißt

Deutsches Autorecht

Der Betrieb

derselbe

Reichsgesetz über die Devisenbewirtschaftung

Deutsche Justiz

Deutscher Juristentag

Die Öffentliche Verwaltung

Deutsche Richterzeitung

Deutsches Steuerrecht

Deutsche Steuer-Zeitung

Deutsches Verwaltungsblatt

European Economic Community

Einführungsgesetz zur Strafprozeßordnung

Vertrag über die Gründung der Europäischen Wirtschaftsgemeinschaft

Einführung

Einleitung

Europäische Konvention zum Schutz der Menschenrechte und Grundfreiheiten

Gerichtshof der Europäischen Gemeinschaften

Entscheidungen des Gerichtshofes der Europäischen Gemeinschaften

Europäische Zeitschrift für Wirtschaftsrecht

Vertrag zur Gründung der Europäischen Wirtschaftsgemeinschaft

fortfolgend

Frankfurter Kommentar zum Gesetz gegen Wettbewerbsbeschränkungen

Fußnote

folgend

Festschrift

Goltdammer's Archiv für Strafrecht

gemäß

Gesetz betreffend die Gesellschaften mit beschränkter Haftung

Gewerbearchiv

Gewerbeordnung

Grundgesetz

Gemeinschaftskommentar zum Gesetz gegen Wettbewerbsbeschränkungen 


\begin{tabular}{|c|c|}
\hline GRUR & Gewerblicher Rechtsschutz und Urheberrrecht \\
\hline GS & Der Gerichtssaal \\
\hline GVG & Gerichtsverfassungsgesetz \\
\hline GWB & Gesetz gegen Wettbewerbsbeschränkungen \\
\hline h.M., H.M. & herrschende Meinung \\
\hline HandwO & Handwerksordnung \\
\hline HGB & Handelsgesetzbuch \\
\hline HGrG & Haushaltsgrundsätzegesetz \\
\hline Hrsg. & Herausgeber \\
\hline Hs. & Halbsatz \\
\hline HWiSTR & Handwörterbuch des Wirtschafts- und Steuerstrafrechts \\
\hline i.d.F. & in der Fassung \\
\hline i.d.S. & in diesem Sinn, in dem Sinne \\
\hline i.Erg. & im Ergebnis \\
\hline i.S. & im Sinne \\
\hline i.V.m. & in Verbindung mit \\
\hline JA & Juristische Arbeitsblätter \\
\hline JGG & Jugendgerichtsgesetz \\
\hline JR & Juristische Rundschau \\
\hline JStG & Jahressteuergesetz \\
\hline Jura & Juristische Ausbildung \\
\hline JuS & Juristische Schulung \\
\hline JW & Juristische Wochenschrift \\
\hline $\mathrm{JZ}$ & Juristenzeitung \\
\hline KartG & Schweizerisches Kartellgesetz \\
\hline KG & Kammergericht \\
\hline KKOWiG & Karlsruher Kommentar zum Ordnungswidrigkeitengesetz \\
\hline KritV & $\begin{array}{l}\text { Kritische Vierteljahreszeitschrift für die Gesetzgebung und } \\
\text { Rechtswissenschaft }\end{array}$ \\
\hline L/R & Löwe/Rosenberg, Kommentar zur StPO \\
\hline LBG/B-W & Landesbeamtengesetz Baden-Württemberg \\
\hline LK & Leipziger Kommentar zum StGB \\
\hline LOWiG & Landesordnungswidrigkeitengesetz \\
\hline m.N. & mit Nachweisen \\
\hline m.w.N. & mit weiteren Nachweisen \\
\hline MDR & Monatsschrift für Deutsches Recht \\
\hline M-G & Müller-Gugenberger \\
\hline Millrd. & Milliarde \\
\hline MOG & Gesetz zur Durchführung der gemeinsamen Marktorganisation \\
\hline NJW & Neue Juristische Wochenschrift \\
\hline Nr. & Nummer \\
\hline NSDAP & Nationalsozialistische Deutsche Arbeiterpartei \\
\hline NStZ & Neue Zeitschrift für Strafrecht \\
\hline NVwZ & Neue Zeitschrift für Verwaltungsrecht \\
\hline NZV & Neue Zeitschrift für Verkehrsrecht \\
\hline ÖJZ & Österreichische Juristenzeitung \\
\hline
\end{tabular}




\begin{tabular}{|c|c|}
\hline OLAF & Europäische Betrugsbekämpfungsorganisation \\
\hline OLG & Oberlandesgericht \\
\hline ord. & Ordonnance \\
\hline öSTGB & Strafgesetzbuch Österreichs \\
\hline OWiG & Gesetz über Ordnungswidrigkeiten \\
\hline RAO & Reichsabgabenordnung \\
\hline $\mathrm{RdF}$ & Reichsministerium der Finanzen \\
\hline RdErl. & Runderlaß \\
\hline Ref. & Referat \\
\hline Rev. int. d. droit pen. & Revue internationale de droit pénale \\
\hline RG & Reichsgesetz \\
\hline RGB1. & Reichsgesetzblatt \\
\hline RiStBV & Richtlinien für das Straf- und Bußgeldverfahren Rnd.Randnummer \\
\hline RobbenG & Gesetz zur Erhaltung der antarktischen Robben \\
\hline RRH & Rebmann/Roth/Hermann, Kommentar zum OWiG \\
\hline Rspr. & Rechtsprechung \\
\hline \multirow[t]{2}{*}{ RsprEinhG } & Gesetz zur Wahrung der Einheitlichkeit der Rechtsprechung der obersten \\
\hline & Gerichtshöfe des Bundes \\
\hline RStBl. & Reichssteuerblatt \\
\hline RVO & Reichsversicherungsordnung \\
\hline RWiG & Reichswirtschaftsgesetz \\
\hline RZB1. & Reichszollblatt \\
\hline S. Ct. & Supreme Court Reporter \\
\hline S. & Seite (in Verbindung mit $\S=$ Satz) \\
\hline SDÜ & Schengener Durchführungsabkommen \\
\hline SeeHeimTportG & $\begin{array}{l}\text { Gesetz betreffend die Verpflichtung der Kauffahrtschifferei zur Mitnahme } \\
\text { heimzuschaffender Seeleute }\end{array}$ \\
\hline SGB & Sozialgesetzbuch \\
\hline SJZ & Süddeutsche Juristenzeitung \\
\hline SK & Systematischer Kommentar zum Strafgesetzbuch \\
\hline sog. & sogenannt(e) \\
\hline S-S & Schönke/Schröder, Kommentar zum Strafgesetzbuch \\
\hline StGB & Strafgesetzbuch \\
\hline StPO & Strafprozeßordnung \\
\hline StV & Strafverteidiger \\
\hline StVG & Straßenverkehrsgesetz \\
\hline StVO & Straßenverkehrsordnung \\
\hline StVZO & Straßenverkehrszulassungsordnung \\
\hline SubvG & Subventionsgesetz \\
\hline TB & Tätigkeitsberichte \\
\hline TierSchG & Tierschutzgesetz \\
\hline u.a. & unter anderem \\
\hline U.S. & United States Reporter \\
\hline U.S.C. & United States Code \\
\hline US & United States \\
\hline UWG & Gesetz gegen den unlauteren Wettbewerb \\
\hline
\end{tabular}




\begin{tabular}{|c|c|}
\hline Verf. & Verfasser \\
\hline VerwArch & Verwaltungsarchiv \\
\hline VerwStrG & Verwaltungsstrafgesetz Schweiz \\
\hline VFSlg. & $\begin{array}{l}\text { Sammlung der Erkenntnisse und wichtigsten Beschlüsse des } \\
\text { Verfassungsgerichtshofes, Neue Folge }\end{array}$ \\
\hline VGH & Verwaltungsgerichtshof \\
\hline Vgl., vgl. & vergleiche \\
\hline VO & Verordnung \\
\hline Vol. & Volume \\
\hline v. & von, vom \\
\hline Vor. & Vorbemerkung \\
\hline VRS & Verkehrsrechtssammlung \\
\hline vs. & versus \\
\hline VStG & Verwaltungsstrafgesetz Österreichs \\
\hline VwGH & Verwaltungsgerichtshof Östereichs \\
\hline VwGO & Verwaltungsgerichtsordnung \\
\hline VwSlg. & $\begin{array}{l}\text { Erkenntnisse und Beschlüsse des Verwaltungsgerichtshofes, } \\
\text { Neue Folge }\end{array}$ \\
\hline VwVfG & Verwaltungsverfahrensgesetz \\
\hline WHG & Wasserhaushaltsgesetz \\
\hline WiGBl. & Gesetzblatt der Verwaltung des Vereinigten Wirtschaftsgebietes \\
\hline WiKG & Gesetz zur Bekämpfung der Wirtschaftskriminalität \\
\hline WiStG 49 & Wirtschaftsstrafgesetz \\
\hline wistra & Zeitschrift für Wirtschaft, Steuer, Strafrecht \\
\hline WuW & Wirtschaft und Wettbewerb \\
\hline WZG & Warenzeichengesetz \\
\hline z.B. & zum Beispiel \\
\hline z.T. & zum Teil \\
\hline ZfW & Zeitschrift für Wasserrecht \\
\hline ZHR & Zeitschrift für das gesamte Handelsrecht und Wirtschaftsrecht \\
\hline Ziff. & Ziffer \\
\hline zit. & zitiert \\
\hline ZLR & Zeitschrift für das gesamte Lebensmittelrecht \\
\hline ZPO & Zivilprozeßordnung \\
\hline ZRP & Zeitschrift für Rechtspolitik \\
\hline ZSR & Zeitschrift für Schweizerisches Recht \\
\hline ZStW & Zeitschrift für die gesamte Strafrechtswissenschaft \\
\hline zust. & zustimmend \\
\hline zutr. & zutreffend \\
\hline zw. & zweifelnd \\
\hline
\end{tabular}

